\title{
Re: Final Report of the Intergroup Randomized Study of Combined Androgen- Deprivation Therapy Plus Radiotherapy Versus Androgen-Deprivation Therapy Alone in Locally Advanced Prostate Cancer
}

\author{
Malcolm D. Mason1, Wendy R. Parulekar2, Matthew R. Sydes3, Michael Brundage4, \\ Peter Kirkbride5, Mary Gospodarowicz6, Richard Cowan7, Edmund C. Kostashuk8, \\ John Anderson9, Gregory Swanson 10, Mahesh K.B. Parmar3, Charles Hayter11, Gordana Jovic3, \\ Andrea Hiltz2, John Hetherington11, Jinka Sathya11, James B.P. Barber14, Michael McKenzie15, \\ Salah El-Sharkawi16, Luis Souhami17, P.D. John Hardman18, Bingshu E. Chen2, Padraig Warde6 \\ ${ }_{1}^{1}$ Cardiff University School of Medicine, Velindre Hospital, London, United Kingdom
}

2NCIC Clinical Trials Group, Queen's University, Kingston, Canada

3 Medical Research Council Clinical Trials Unit at University College London, London, United Kingdom

${ }^{4}$ Cancer Centre of Southeastern Ontario, Kingston, Canada

5The Clatterbridge Cancer Centre National Health Service Foundation Trust, Wirral, United Kingdom

6 University of Toronto, Princess Margaret Cancer Centre, Toronto, Canada

7 Christie Hospital, University of Manchester, Manchester, United Kingdom

8 Fraser Valley Cancer Centre, Surrey, Canada

9Sheffield Teaching Hospitals, National Health Service Foundation Trust, Sheffield, United Kingdom

10University of Texas Health Science Center, San Antonio, TX, USA

11 University of Toronto, Carlo Fidani Peel Regional Cancer Center, Toronto, Canada

12 Castle Hill Hospital, Hull, United Kingdom

13Memorial University of Newfoundland, St Johns, Newfoundland and Labrador, Canada

14Velindre Hospital, Cardiff, United Kingdom

15 Vancouver Cancer Centre, Vancouver, British Columbia

16South West Wales Cancer Centre, Swansea, United Kingdom

17 McGill University, Montreal, Quebec, Canada

18The James Cook University Hospital, Middlesbrough, United Kingdom

J Clin Oncol 2015 Feb 17. pii: JC0.2014.57.7510. [Epub ahead of print]

\section{EDITORIAL COMMENT}

No certain treatment recommendations were given for locally advanced or high-risk prostate cancer in the European Association of Urology (EAU) guidelines (1). In the guidelines, studies supporting surgery or radiotherapy (RT) were listed, and the readers were left alone to make their own decisions.

In the present study, Mason et al. reported the impact of adding RT to androgen deprivation therapy (ADT). One thousand two hundred and five patients with T34, No/Nx, M0 prostate cancer or T1-2 disease with either PSA more than $40 \mu \mathrm{g} / \mathrm{L}$ or PSA 20 to $40 \mu \mathrm{g} / \mathrm{L}$ plus Gleason score of 8 to 10 were randomized to ADT alone $(n=602)$ or to ADT+RT ( $n=603)$. A lower dose radiation 64 to 69 Gy was used for RT. Overall survival (OS) risk reduction was $30 \%$ for $A D T+R T$ group ( $<<0.001)$ at a median follow-up of 8 years. Cancer-specific survival (CSS) was significantly improved by the addition of RT to ADT (HR: $0.46,95 \%$ Cl: 0.34 to $0.61 ; p<0.001$ ). Patients on ADT+RT reported a higher frequency of adverse events related to bowel toxicity. However, reported frequency of ADT-related toxicities (impotence, hot flushes, urinary frequency, ischemia, and hypertension) were similar for both arms. The present study provided results of high-risk patients in a longer median follow-up time than SPCG-7 study (2). Because the study took place between 1995 and 2005, less than 70 Gy was used for RT. Even at lower radiation doses, the authors confirmed that adding RT to ADT improved both OS and cancer-specific survival (CSS) with minimal general toxicity. In the modern era, improved RT techniques may help achieve better outcomes with much higher radiation doses without increased morbidity in this group of patients.

\section{REFERENCES}

1. Heidenreich A, Bastian PJ, Bellmunt J, Bolla M, Joniau S, van der Kwast T, Mason M, Matveev V, Wiegel T, Zattoni F, Mottet N. EAU guidelines on prostate cancer. Part II: Treatment of advanced, relapsing, and castration-resistant prostate cancer. Eur Urol. 2014;65:467-479.

2. Widmark A, Klepp O, Solberg A, Damber JE, Angelsen A, Fransson P, Lund JA, Tasdemir I, Hoyer M, Wiklund F, Fosså SD. Endocrine treatment, with or without radiotherapy, in locally advanced prostate cancer (SPCG-7/SFU0-3): an open randomised phase III trial. Lancet 2009;373:301-308. 